\title{
Transcriptional changes induced by bevacizumab combination therapy in responding and non-responding recurrent glioblastoma patients
}

Thomas Urup ${ }^{*}$, Line Mærsk Staunstrup², Signe Regner Michaelsen ${ }^{1}$, Kristoffer Vitting-Seerup ${ }^{2}$, Marc Bennedbæk ${ }^{3}$, Anders Toft ${ }^{1}$, Lars Rønn Olsen ${ }^{4,5}$, Lars Jønson ${ }^{3}$, Shohreh Issazadeh-Navikas ${ }^{6}$, Helle Broholm ${ }^{7}$, Petra Hamerlik ${ }^{1,8}$, Hans Skovgaard Poulsen ${ }^{1,9}$ and Ulrik Lassen ${ }^{1,9,10}$

\begin{abstract}
Background: Bevacizumab combined with chemotherapy produces clinical durable response in $25-30 \%$ of recurrent glioblastoma patients. This group of patients has shown improved survival and quality of life. The aim of this study was to investigate changes in gene expression associated with response and resistance to bevacizumab combination therapy.

Methods: Recurrent glioblastoma patients who had biomarker-accessible tumor tissue surgically removed both before bevacizumab treatment and at time of progression were included. Patients were grouped into responders $(n=7)$ and non-responders $(n=14)$. Gene expression profiling of formalin-fixed paraffin-embedded tumor tissue was performed using RNA-sequencing.
\end{abstract}

Results: By comparing pretreatment samples of responders with those of non-responders no significant difference was observed. In a paired comparison analysis of pre- and posttreatment samples of non-responders 1 gene was significantly differentially expressed. In responders, this approach revealed 256 significantly differentially expressed genes (72 down- and 184 up-regulated genes at the time of progression). Genes differentially expressed in responders revealed a shift towards a more proneural and less mesenchymal phenotype at the time of progression.

Conclusions: Bevacizumab combination treatment demonstrated a significant impact on the transcriptional changes in responders; but only minimal changes in non-responders. This suggests that non-responding glioblastomas progress chaotically without following distinct gene expression changes while responding tumors adaptively respond or progress by means of the same transcriptional changes. In conclusion, we hypothesize that the identified gene expression changes of responding tumors are associated to bevacizumab response or resistance mechanisms.

Keywords: Anti-angiogenic, RNA-sequencing, Protein kinase C, Reverse mesenchymal transition, TGF-beta

\footnotetext{
* Correspondence: thomas.urup@regionh.dk

'Department of Radiation Biology, The Finsen Center, Section 6321,

Rigshospitalet, Blegdamsvej 9, DK-2100 Copenhagen, Denmark

Full list of author information is available at the end of the article
} 


\section{Background}

Glioblastoma is the most malignant primary brain tumor in adults. Standard treatment comprises surgery followed by radio-chemotherapy with concomitant and adjuvant temozolomide. Despite this aggressive treatment the prognosis is dismal with a median survival of 14.6 months [1]. Recurrence of glioblastoma is almost inevitable and at recurrence numerous agents have shown limited clinical effect [2].

Glioblastoma is characterized by excessive and aberrant angiogenesis. Anti-angiogenic agents inhibiting vascular endothelial growth factor A (VEGF) have been shown to normalize the tumor vasculature and improve blood flow and drug delivery $[3,4]$. This emphasizes the potential value of combining anti-angiogenic therapy with drugs targeting the tumor. Bevacizumab, a VEGF targeting antibody, in combination with chemotherapy is among the most frequently used treatments in recurrent glioblastoma patients. Although this treatment regimen has not proved active in the total population of recurrent glioblastoma patients [5], 25-30\% of the patients achieve treatment response (defined as radiological and clinical improvement). This group of patients has demonstrated improved survival as well as quality of life [6-9], highlighting the importance of identifying predictive biomarkers for bevacizumab efficacy.

Glioblastoma consists of a mixture of cancer cell subclones, glial cells, stromal cells and immune cells, and each of these cell populations adds to the tumor heterogeneity. This complicates the interpretation of tumor biomarker analysis. Nevertheless, gene expression profiling of glioblastoma has identified four molecular subtypes, namely Neural, Proneural, Classical and Mesenchymal, and preliminary evidence indicates survival benefit in distinct molecular subtypes treated with bevacizumab combination therapy $[10,11]$. However, the results of these two studies have been inconsistent and we have along with others shown that the subtypes do not impact bevacizumab response in recurrent glioblastoma [12, 13].

Due to the rarity of paired, biomarker evaluable, recurrent glioblastoma tissue samples, our current knowledge on bevacizumab response and resistance mechanisms is based on preclinical animal studies and small clinical case reports [14-19]. Recently, novel gene expression technologies, including RNA-sequencing (RNA-Seq), have shown high performance on formalin-fixed paraffin embedded (FFPE) glioblastoma samples $[10,11]$. This will prove valuable for future clinical biomarker studies on archived tumor tissue.

In this study, we hypothesized that bevacizumab combination treatment exerts selective pressure on the tumors and creates adaptive transcriptional changes as tumors respond and progress. Accordingly, the aim was to identify transcriptional changes by RNA-Seq in paired tumor samples, before and after bevacizumab treatment in both responding and non-responding recurrent glioblastoma patients.

\section{Methods \\ Patients}

All patients with glioblastoma (pathologically confirmed WHO grade IV) treated at recurrence with bevacizumab plus irinotecan at Rigshospitalet in the period between May 2005 and December 2014, were assessed for eligibility. Eligibility criteria for this study were 1) response evaluability and 2) biomarker accessible tumor tissue prior to bevacizumab treatment and at time of progression after bevacizumab treatment. The criteria are specified below.

\section{Treatment and clinical follow-up}

Treatment at recurrence followed Danish national guidelines and was planned at a multidisciplinary team conference. If the neurosurgeons considered the tumor amenable for relapse surgery, this was offered in order to remove as much tumor tissue as possible. Bevacizumab and irinotecan therapy was administered according to a published treatment protocol [20]. Prior to initiation of treatment the patients had to have measurable progressive disease by contrast-enhanced MRI after standard therapy and had to be at least 4 weeks from prior chemotherapy and 3 months from completion of radiation therapy. Clinical and radiological follow-up was performed according to protocol [20]. Treatment response was evaluated based on the RANO criteria and response was confirmed on the subsequent follow-up MRI [21]. Responders were defined as patients with complete or partial response $(C R+P R)$ and non-responders were defined as patients with stable disease (SD) or progressive disease (PD).

\section{Sample acquisition and RNA preparation}

A total of 264 patients were assessed for eligibility. Twenty-four response-evaluable patients had surgery before and after bevacizumab treatment and had archived paired FFPE tissue blocks at the Department of Pathology, Rigshospitalet. Tissue review was conducted by a neuropathologist, who was blinded to clinical outcome. The number of tumor cells was estimated based on hematoxylin and eosin-staining. Macrodissection was performed in a few cases to remove large amounts of normal brain tissue and only samples containing a tumor cell frequency $>50 \%$ were selected for RNA-extraction. If tumor blocks from relapse surgery prior to bevacizumab treatment were available and contained sufficient amount of tumor cells, they were included in preference to tumor blocks from time of glioblastoma diagnosis. All postbevacizumab samples were obtained from relapse surgery following progression on bevacizumab treatment and no intermediate relapse therapy was administered. Three patients had an insufficient number of tumor cells in one of 
the paired tumor blocks and were excluded prior to analysis. Thus, a total of 21 patients with paired tumor blocks were included in the study. Samples were sectioned into $3 \times 10 \mu \mathrm{m}$ thick FFPE sections and RNA was extracted from paired tumor blocks in three equal sample-sized batches using Deparaffinization Solution (Qiagen, Ca. No. 19093) and RNeasy FFPE kit (Qiagen, Ca. No. 73504). RNA extracts were stored at $-80^{\circ} \mathrm{C}$.

\section{Library preparation for RNA-sequencing}

Library preparation was carried out using the strandspecific Ovation Human FFPE RNA-Seq Library Systems from Nugen according to the instructions from the manufacturer. $250 \mathrm{ng}$ of total RNA was used as input material for the cDNA synthesis and the double stranded cDNA was fragmented on the Covaris S2 (Covaris, Inc.) in microtubes using the following settings: duty cycle-10\%/Intensity-5/cycles/burst-200 for a total of $180 \mathrm{~s}$. RNA-Seq was performed on Illumina HiSeq 2500 (Illumina) as paired end sequencing $2 \times 101$ bases in Rapid Mode with 5 samples per run resulting in approximately 175 million paired-end reads per run. Raw data was converted to fastq files using CASAVA v1.8.2.

\section{Filtering and differential expression analysis}

Detailed description of the RNA-Seq analysis can be found in Additional file 1: Supplementary Methods. Briefly, the raw sequencing data was trimmed with
Trimmomatic and mapped to the human genome ( $h g 19)$ with TopHat2 [22, 23]. Genes were annotated (Ensemble annotation, release 66) and expression levels were quantified using featureCounts [24]. Libraries with less than $10 \%$ of genes having more than 15 fragments were discarded $(n=6)$. Only genes with at least 10 fragments and an abundance of at least 3 Fragments Per Kilobase per Million reads (FPKM) in at least 5 libraries in any of the 4 patient-groups (Fig. 1) were kept for further analysis (15,630 genes). Differential expression analysis was performed using edgeR (v. 3.12.0) either as paired analysis (comparison 1 and 3 ) or a batch-corrected analysis (comparison 2, see Fig. 1). P-values were corrected for multiple testing using the False Discovery Rate (FDR) approach and genes with adjusted $P$-values $<0.05$ were considered significant. Analysis was performed using the software $\mathrm{R}$ version 3.2.2 ( $\mathrm{R}$ Development Core Team, Vienna, Aurstria, http://www.R-project.org). Expression data are available at the NCBI Geo datasets, accession number GSE79671.

\section{Gene set enrichment analysis}

Gene ontology gene-sets were downloaded (6th Jan 2016) from The European Bioinformatics Institute's official Gene Ontology mirror. Gene ontology terms from the 5th level of the hierarchical gene ontology term tree were used. Gene sets c2, c3, c6 and $\mathrm{H}$ were downloaded from The Molecular Signatures Database (MSigDB [25],

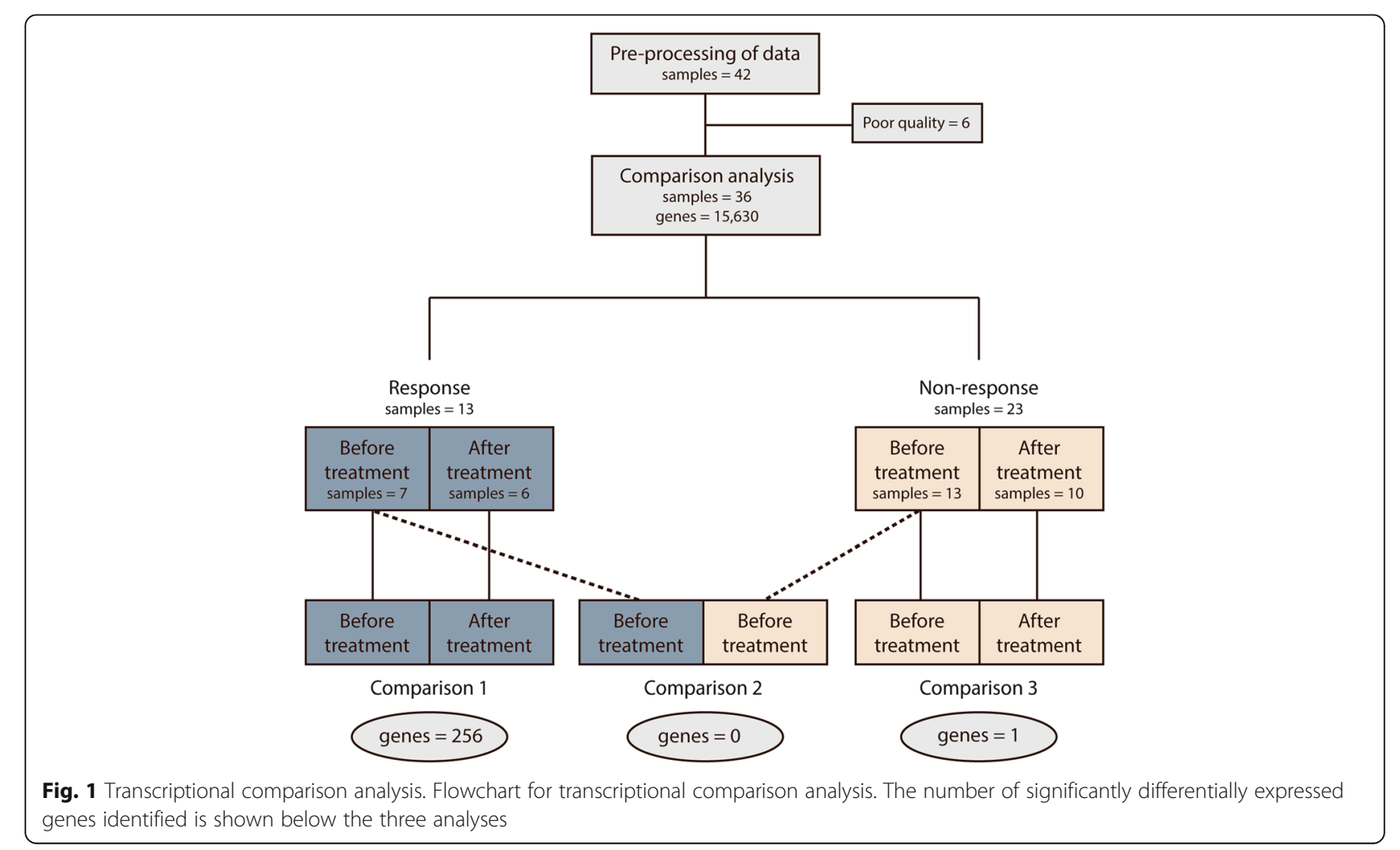


via http://bioinf.wehi.edu.au/software/MSigDB/). The enrichment analysis was done using a Fisher's exact test only considering the 15,630 tested genes, and $P$-values were FDR corrected and adjusted $P$-value $<0.05$ were considered significant.

\section{Ingenuity pathway analysis}

Differentially expressed genes were analyzed by QIAGEN's Ingenuity Pathway Analysis (IPA) using the core analysis with default settings and 15,630 tested genes from the RNA-Seq dataset as background (IPA ${ }^{\circ}$,QIAGEN Redwood City, www.qiagen.com/ingenuity). The software uses a large database of curated data and computes a score for each network according to the fit of the set of genes supplied in the analysis. The scores were calculated by right-tailed Fisher's exact test. The scores derived from $P$-values, indicate the likelihood of supplied genes belonging to a network versus those obtained by chance. A consistency score (Z-score) $>2$ or $<-2$ indicates with $\geq 99 \%$ confidence that a supplied gene network was not generated by chance alone. Enrichment of "canonical pathways" and "up-stream regulators" with a Z-score $>2$ or < -2 were considered for analysis [26].

\section{Results}

\section{Patient characteristics}

Patient characteristics and clinical outcome are shown in Table 1. All patients had received standard treatment with radio- and chemotherapy (temozolomide) prior to bevacizumab combination therapy. All patients had undergone relapse surgery prior to bevacizumab combination therapy but only half of the resected tumor samples were eligible for biomarker analysis. Consequently, the remaining half of the samples obtained before bevacizumab therapy was from surgery before standard treatment. Patient and sample characteristics did not differ significantly between responders and non-responders. Responders had a significantly longer progression-free survival compared to non-responders $(P=0.02)$ while no significant difference was observed in overall survival $(P=0.16)$.

Of the 42 samples, high quality RNA-Seq data was obtained on a total of 36 samples, leaving 20 "prebevacizumab samples" and 16 "post-bevacizumab samples" and 16 paired samples. Of the paired samples, 6 patients were classified as responders and 10 patients were classified as non-responders.

\section{Group comparisons of gene expression profiles}

To identify significantly differentially expressed genes between groups, comparison analyses were performed according to a pre-specified analytical strategy, shown in Fig. 1. In the analyses, no confounding effects of clinical factors and no genetic subgroups were identified.

The comparison of pretreatment samples between responders $(n=7)$ and non-responders $(n=13)$ demonstrated no significantly differentially expressed genes.

To identify transcriptional changes at the time of progression compared to before treatment a paired analysis was performed in non-responders $(n=10)$ and responders $(n=6)$, separately: In non-responders, 1 gene was significantly upregulated at the time of progression (Additional file 2: Table S1). In responders, a total of 256 genes were found significantly differentially expressed, including 72 downregulated and 184 upregulated genes at the time of progression (Additional file 3: Table S2 and Additional file 4: Table S3).

To analyze if the larger number of patients in the non-response group explained the absence of significant genes we performed a subsampling analysis. This analysis subsampled pairs of non-responders to random groups of 6 patients (100 times) and here we found that the mean number of differentially expressed genes (mean: 2.6; range: 0-33) was approximately 100 times lower than the number of significantly differentially expressed genes found in responders (Additional file 5: Figure S1), indicating the results are not due to differences in sample sizes.

Collectively, we were not able to identify differentially expressed genes between pretreatment samples of responders and non-responders. Furthermore, bevacizumab combination therapy produced a significant impact on the transcriptional changes in responders at time of progression, but only minimal changes in non-responders.

\section{Functional analysis of transcriptional changes in responders}

In contrast to the non-protein coding gene (small nucleolar RNA, H/ACA box 22; SNORA22) identified in non-responders, several of the 256 genes identified in responders have been functionally well-characterized in published literature. To identify functional mechanisms related to the gene expression changes in responders, the Molecular Signatures Database (MSigDB) was used to find gene ontologies and gene lists significantly enriched by the up- and down-regulated genes. The top-10 most significantly enriched gene ontologies and gene lists are shown in Additional file 6: Table S4 and Additional file 7: Table S5.

Gene ontology analysis showed that the upregulated genes are implicated in nervous system development, neuron signaling and neuron differentiation. Down-regulated genes are involved in blood 
Table 1 Patient characteristics

$\begin{array}{llll}\text { All patients } & \text { Responders } & \text { Non- } & \text {-value } \\ (n=21) & (n=7) & \text { responders } \\ (n=14) & \\ & \end{array}$

Gender, $n(\%)$

$11(52)$

$5(71)$

$6(43)$

0.36

Female

$10(48)$

2 (29)

8 (57)

Age, years (range)

Median

$52(21-70)$

$53(35-65)$

$48(21-70)$

0.66

WHO performance status, $n(\%)$

0

$10(48)$

2 (29)

4 (57)

1 (14)

Secondary glioblastoma, n (\%)

$$
\text { Yes }
$$

No

$1(5)$

20 (95)

Standard glioblastoma therapy,

$n(\%)$

Yes

No

Prior lines of chemotherapy, $n(\%)$

$$
\begin{aligned}
& 1 \\
& 2
\end{aligned}
$$

Tumor size, $\mathrm{cm}^{2}$ (range)

$$
\text { Median }
$$

7 (100)

0

18 (86)

$7(100)$

$9(1-28)$

$11(4-28)$

Multifocal disease, $n$ (\%)

Yes

$2(10)$

19 (91)

14 (67)

7 (33)

8 (38)

$13(62)$

0

7 (100)

Corticosteroid use, $n(\%)^{a}$

Yes

No

Neurocognitive deficit, $n$ (\%)

Yes

No

Primary sample, before bevacizumab, $n$ (\%)

Initial glioblastoma diagnosis

Relapse surger
bevacizumab

Time duration from relapse surgery (after bevacizumab), months

to initiation of standard therapy, median

to last bevacizumab administration, median

Number of bevacizumab treatment cycles ${ }^{b}$

Median
8 (57)

5 (36)

1 (7)

$13(93)$

1.00

1 (7)

11 (79)

0.52

$3(21)$ 
Table 1 Patient characteristics (Continued)

\begin{tabular}{|c|c|c|c|c|}
\hline \multicolumn{5}{|l|}{$\begin{array}{l}\text { Bevacizumab combination } \\
\text { therapy, } n(\%)\end{array}$} \\
\hline Irinotecan & $17(81)$ & $6(86)$ & $11(79)$ & 1.00 \\
\hline Irinotecan and cetuximab & $4(19)$ & $1(14)$ & $3(21)$ & \\
\hline \multicolumn{5}{|l|}{ Response, $n(\%)$} \\
\hline Response (CR + PR) & $7(33)$ & $7(100)$ & 0 & \\
\hline Stable disease & $10(48)$ & 0 & $10(71)$ & \\
\hline Progressive disease & $4(19)$ & 0 & $4(29)$ & \\
\hline \multicolumn{5}{|l|}{$\begin{array}{l}\text { Progression-free survival, } \\
\text { months }\end{array}$} \\
\hline Median & 5.4 & 10.8 & 3.9 & 0.02 \\
\hline \multicolumn{5}{|l|}{ Overall survival, months } \\
\hline Median & 10.8 & 14.3 & 8.6 & 0.16 \\
\hline
\end{tabular}

Abbreviations: $C R$ complete response, $P R$ partial response

a Prednisolone $>10 \mathrm{mg}$

${ }^{b}$ Two bevacizumab combination treatments ( 28 days) defined one treatment cycle

vessel development, collagen metabolism and endodermal differentiation.

Among the gene lists significantly enriched by the upregulated genes are three with high density of CpGpromoters bearing histone $\mathrm{H} 3$ trimethylation mark at K27 (H3K27me3). The gene list most significantly enriched by the down-regulated genes characterizes epithelial-mesenchymal transition. Interestingly, the mesenchymal and proneural subtypes defined by Verhaak overlapped significantly with the downregulated and up-regulated genes, respectively.

Collectively, this analysis shows that responding glioblastomas when progressing express reduced levels of angiogenesis-related genes and higher levels of genes involved in neuronal development and signaling. Furthermore, the gene profiles changed towards a less mesenchymal phenotype and more proneural subtype at progression.

\section{Dynamical changes in molecular subtype profiles}

To investigate if bevacizumab treatment affects the expression of genes defining the molecular subtypes, gene expression in the paired samples of responders were analyzed according to subtype gene lists [11, 27]. As shown in Fig. 2, we observed that genes defining the Verhaak classical subtype were almost equally up- and downregulated, while the majority of mesenchymal genes were down-regulated at the time of progression. In contrast, most of the neural and proneural genes were upregulated at progression. According to the adapted Phillips classifier all genes of the mesenchymal subtype

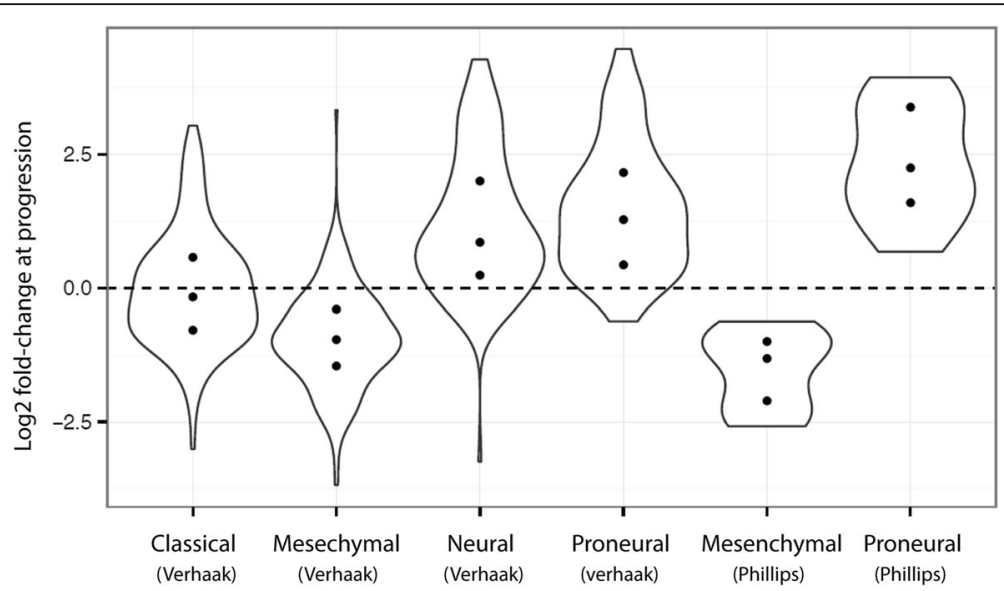

Fig. 2 Expression of genes defining molecular subtypes. Paired gene expression fold-changes of genes defining molecular subtypes at the time of progression compared to before initiation of bevacizumab therapy in responding patients. $\bullet$ indicates the gene expression change according to the $25 \%$ percentile of subtype genes. ${ }^{*}$ Modified Phillips classifier used on the AVAglio dataset 
were down-regulated and all genes of the proneural subtype were up-regulated at progression.

\section{Ingenuity pathway analysis of transcriptional changes in responders}

In order to investigate the structure of possible regulatory networks underlying the significant gene expression changes in responders, we used the IPA. Unlike the enrichment analysis, IPA allows identification of biological networks, including gene relationships and interactions, linked to specific known biological functions or pathways.

First, a canonical pathway analysis was performed to find activated or inhibited pathways. "Integrin signaling" was the only significantly inhibited pathway (Additional file 8: Table S6). Fifteen canonical pathways were found activated and one of these involved "calcium signaling", while the remaining 14 pathways all included protein kinase $\mathrm{C}$ related signaling genes (PRKCB, PRKCE, PRKCZ and others).

The IPA analysis identified 2 activated (estrogen receptor and SPDEF) and 4 inhibited (TGF- $\beta 1$, SMAD3, ERK and ERRB2) upstream regulators (Additional file 9: Table S7). Out of the 6 upstream regulators, TGF- $\beta 1$ was the most significant (Z-score $=-4.0)$ and TGFB1 was the only gene which, based on our RNA-Seq data, trended toward a down-regulation in responders (raw $P=0.006$; adjusted $P=0.15 ; \log 2$ fold-change $=-1.08)$ while this was not observed in non-responders (raw $P=0.57$ ). Consequently, we focused on TGF- $\beta 1$ and by using the mechanistic network function in IPA, we generated a plausible directional network from TGF- $\beta 1$ and its closest related upstream regulator molecules. As shown in Fig. 3, this network consisted of two inhibited regulators SMAD3, HIF1A and one activated regulator PPARG, of which HIF1A was the only gene trending toward a down-regulation in responders (raw $P=0.03$; adjusted
$P=0.36 ; \log 2$ fold-change $=-0.77)$, while this was not observed in non-responders (raw $P=0.51$ ). These upstream-regulators directly or indirectly induce downstream effector molecules involved in cell-cycle check point regulation $(\mathrm{CDKN} 1 \mathrm{~A})$ and extracellular matrix remodeling (SERPINE-1). These effector molecules in addition to others, shown in Additional file 10: Figure S2, were predominantly found transcriptionally downregulated at the time of recurrence, suggesting that TGF- $\beta 1$ signaling is inactive when tumors progress.

In summary, the pathway analysis showed that protein kinase $C$ signaling was activated in progressing tumors. The analysis found TGF- $\beta 1$ and HIF1A inhibited and a down-regulated trend was confirmed in the RNA-Seq data. These two up-stream regulators are known to regulate extracellular remodeling and cell-cycle, indicating that responding tumors at progression express reduced extracellular matrix remodeling and increased proliferation.

\section{Histological changes}

To investigate a possible association between the identified transcriptional changes and morphological changes of the tumor, we performed a non-blinded review of hematoxylin-eosin stained pre- and posttreatment samples. However, no gross differences or changes were observed in the amount and morphology of tumor cells, stromal cells, neural/glial cells, blood vessels or architecture of the extracellular matrix. Representative images of two responding glioblastomas are shown in Additional file 11: Figure S3.

\section{Discussion}

In this study of recurrent glioblastoma patients, we performed RNA-Seq on tumor tissue surgically removed before and after bevacizumab combination therapy. In
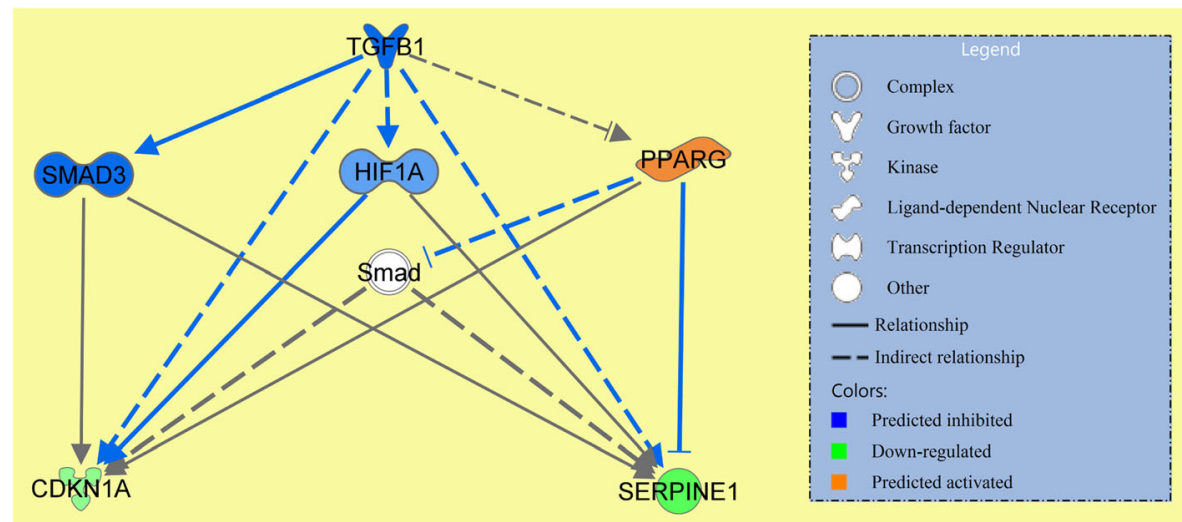

Fig. 3 Mechanistic network of inhibited TGF- $\beta 1$. Generated on the basis of relationships to identified transcriptional changes in responders at the time of progression. The three closest related regulators in the network are SMAD3, HIF1A and PPARG and the two most related downstream molecules are CDKN1A and SERPINE1 
line with others [12], we found no significant differences between pretreatment samples of responders and nonresponders. Considering the extreme inter-tumor heterogeneity of glioblastoma and the small sample size, this was not unexpected. Taking this into account, we pre-specified a paired analytical strategy of before and after treatment samples according to treatment response. The results of this analysis reveal significant transcriptional changes in patients responding to bevacizumab while such changes were almost absent in patients not responding. This suggests that non-responding glioblastomas progress chaotically without following any distinct gene expression changes while responding tumors adaptively respond or progress to bevacizumab treatment by means of the same transcriptional changes.

By functional data mining of published literature, we studied the transcriptional changes of responding glioblastomas to uncover potential response and resistance mechanisms to bevacizumab treatment.

First, it is important to acknowledge that the patients underwent relapse surgery 2 months after bevacizumab treatment cessation. This may explain why no morphological changes in the vasculature were observed, as blood vessels can grow and remodel extensively within few weeks [28]. Nevertheless, gene ontology analysis found angiogenesis related genes significantly downregulated at the time of relapse. The reason for these conflicting findings remains unexplained.

The enrichment analysis revealed that up-regulated genes at time of progression were significantly overrepresented by genes involved in neural development and differentiation processes. Furthermore, up-regulated genes were significantly enriched by genes that are known to be up-regulated due to de-methylation of H3K27 promoter regions - a process which is known to be related to decreased activity of Polycomp Repressive Complex 2 (PRC2) during differentiation [29]. Accordingly, epigenetic regulation may be associated with the up-regulated neural differentiation genes. However, no morphological changes were observed in regards to tumor or stromal cells.

It has previously been found that some glioma patients with recurrent disease after non-bevacizumab treatment shift from a proneural tumor into a mesenchymal subtype [30]. Preclinical glioblastoma studies have shown that adaptive resistance to anti-angiogenic agents is characterized by a transition to a mesenchymal phenotype $[17,18]$. In contrast, we observed that bevacizumab responding glioblastomas shift into a less mesenchymal and more proneural subtype when progressing.

By using the Ingenuity Pathway Analysis software, we identified TGF- $\beta 1$ as the most central up-stream regulator associated with the identified gene expression changes. TGF- $\beta 1$ was found inactivated at time of progression and this was associated with down-regulated extracellular matrix remodeling genes of which several define the mesenchymal subtype signature [27]. This suggests that the shift toward a less mesenchymal phenotype may be related to inactivation of TGF- $\beta 1$ downstream signaling. In line with this finding, it has been shown in preclinical glioblastoma models that TGF- $\beta$ signaling induces a mesenchymal shift, while inhibition of TGF- $\beta$ prevents this shift [31]. Accordingly, the subtypes appear plastic and if the subtypes are representing specific cancer cell lineages, as originally proposed [30], bevacizumab responding glioblastomas may transdifferentiate during progression. Another possibility is that interactions between tumor cells and microenvironment impact subtype classifications, similar to what is seen in epithelial cancers [32]. In this case, bevacizumab induced normalization of the tumor microenvironment and vasculature [33, 34], may change the gene profile accordingly.

Hypoxia has been identified as a central driver of acquired resistance to anti-angiogenic agents in preclinical animal models [16], and hypoxia stimulates secretion of TGF- $\beta$ which can lead to mesenchymal transition [35]. Thus, one could speculate that reduced hypoxia, as a consequence of bevacizumab-induced vascular normalization, may lead to TGF- $\beta$ inhibition and reverse mesenchymal transition. Interestingly, and in line with our results, it has recently been found that breast cancer patients responding to bevacizumab demonstrate reduced levels of tumor hypoxia leading to reduced activity of TGF- $\beta$ [36].

The IPA analysis revealed a significant overrepresentation of genes associated with activated protein kinase $\mathrm{C}$ signaling at the time of relapse. This pathway has a central role in tumor-derived VEGF-induced angiogenesis, and in preclinical tumor models protein kinase $\mathrm{C}$ inhibitors have shown anti-angiogenic activity [37]. Accordingly, protein kinase $C$ mediated VEGF secretion may induce resistance to bevacizumab and may serve as a target in bevacizumab responding glioblastoma patients.

Our study presented a few limitations. The small number of highly selected patients may or may not have introduced selection bias. The lack of paired glioblastomas treated without bevacizumab containing regimens make it difficult to interpret whether the observed effects are related to bevacizumab response or treatment response in general. In this context, the exploratory results have to be carefully interpreted taking these limitations into account. Nevertheless, the baseline patient/sample characteristics and gene profiles did not differ between responding and nonresponding patients and the pre-analytical design of the study make it less likely that the observed changes are independent of response to bevacizumab combination therapy. 


\section{Conclusions}

To our knowledge this is the first study to demonstrate that bevacizumab combination treatment has a significant impact on transcriptional changes in a paired analysis of responding glioblastoma patients. Such changes were minimal in patients not responding. In conclusion, we hypothesize that the identified adaptive changes of bevacizumab responding glioblastomas are related to response or resistance mechanisms. If validated, these data may prove valuable for identification of new and more efficient bevacizumab combination regimens.

\section{Additional files}

Additional file 1: Supplementary methods Analysis of study dataset: Mapping and quantification, Filtering and differential expression, Gene-set analysis (DOCX $26 \mathrm{~kb}$ )

Additional file 2: Table S1. Differentially expressed gene in nonresponders (1 gene) (DOCX $14 \mathrm{~kb}$ )

Additional file 3: Table S2. Down-regulated genes in responders (72 genes) (DOCX $21 \mathrm{~kb}$ )

Additional file 4: Table S3. Up-regulated genes in responders (184 genes) (DOCX $32 \mathrm{~kb}$ )

Additional file 5: Figure S1. Subsampling analysis. This analysis subsampled pairs of non-responders (Comparison 3) to random groups of 6 patients 100 times. Comparison 1 and Comparison 3 shows the number of differentially expressed genes in the paired comparison analysis of responders and non-responders, respectively (DOCX 51 kb)

Additional file 6: Table S4. Gene set enrichment analysis of up-regulated genes (DOCX $17 \mathrm{~kb}$ )

Additional file 7: Table S5. Gene set enrichment analysis of down-regulated genes (DOCX $18 \mathrm{~kb}$ )

Additional file 8: Table S6. Ingenuity Pathway Analysis of activated and inhibited canonical pathways (DOCX $18 \mathrm{~kb}$ )

Additional file 9: Table S7. Ingenuity Pathway Analysis of activated and inhibited up-stream regulators (DOCX $15 \mathrm{~kb}$ )

Additional file 10: Figure S2. Mechanistic network of inhibited TGF- $\beta 1$ and the three most interconnected regulators (SMAD3, HIF1A and PPARG) of downstream molecules identified differentially expressed in responders at the time of progression (DOCX $122 \mathrm{~kb}$ )

Additional file 11: Figure S3. Hematoxylin-eosin staining of two representative responding glioblastomas before and after bevacizumab therapy (DOCX $2297 \mathrm{~kb})$

\section{Abbreviations}

CDKN1A: Cyclin-dependent kinase inhibitor 1A; CR: Complete response; ERK: Extracellular signal-regulated kinase; ERRB2: erb-b2 receptor tyrosine kinase 2; FDR: False discovery rate; FFPE: Formalin-fixed paraffin embedded; FPKM: Fragments per kilobase per million reads; H3K27me3: Histone H3 thimethylation mark at K27; HIF1A: Hypoxia-inducible factor 1-alpha; IPA: Ingenuity pathway analysis; MRI: Magnetic resonance imaging; MSigDB: Molecular signatures database; PD: Progressive disease PPARG: Peroxisome proliferator-activated receptor gamma; PR: Partial response; PRC2: Polycomp repressive complex 2; PRKCB: Protein kinase C, beta; PRKCE: Protein kinase C, epsilon; PRKCZ: Protein kinase C, zeta; RANO: Response assessment in neuro-oncology criteria; RNA-seq: RNA sequencing; SD: Stable disease; SERPINE-1: Serine protease inhibitor 1; SPDEF: Sam pointed domain containing ETS transcription factor; TGF$\beta 1$ : Transforming growth factor beta 1; VEGF: Vascular endothelial growth factor

\section{Acknowledgements}

Not applicable.

\section{Funding}

The study was kindly supported by Rigshospitalet, the Danish Cancer Society, Doctor Sophus Carl Emil Friis and Wife Olga Friis' grant and I.M. Daehnfeldt Foundation. None of the funding bodies were involved in the study design, data collection, analysis, data interpretation or manuscript writing.

\section{Availability of data and material}

The datasets generated and analyzed during the current study are available in the Gene Expression Omnibus (GEO) repository: http://

www.ncbi.nlm.nih.gov/geo/query/

acc.cgi?token=ohqpiosqbdcpboj\&acc=GSE79671

\section{Authors' contributions}

TU, SRM, HSP and UL have contributed with the design of the study, interpretation of data and drafting of manuscript. LMS, KVS, MB, LRO and L have contributed substantially to the bioinformatic analyses and interpretation of data. HB has contributed to sample preparations, histological analyses and manuscript drafting. AT, SIN and PH have contributed substantially to data interpretation, manuscript drafting and revision. All authors edited and approved the final manuscript.

\section{Competing interests}

The authors declare that they have no competing interests.

\section{Consent for publication}

Exemption from obtaining informed consent for publication was granted by the National Ethical Committee (1304518) as patients were either deceased or fatally ill. Patient data were anonymized and de-identified prior to analysis.

\section{Ethics approval and consent to participate}

The study was conducted in accordance with the Helsinki Declaration. The study was approved by the Regional Ethical Committee (H-2-2012-069) and the Danish National Ethical Committee (1304518). Exemption from obtaining informed consent to participate was granted by the National Ethical Committee as patients were either deceased or fatally ill.

\section{Publisher's Note}

Springer Nature remains neutral with regard to jurisdictional claims in published maps and institutional affiliations.

\section{Author details}

${ }^{1}$ Department of Radiation Biology, The Finsen Center, Section 6321, Rigshospitalet, Blegdamsvej 9, DK-2100 Copenhagen, Denmark. ${ }^{2}$ Section for Computational and RNA biology (SCARB), Department of Biology, University of Copenhagen, Ole Maaløesvej 5, DK-2200 Copenhagen, Denmark. ${ }^{3}$ Center for Genomic Medicine, Rigshospitalet, Blegdamsvej 9, DK-2100 Copenhagen, Denmark. ${ }^{4}$ Department of Biology, The Bioinformatics Centre, University of Copenhagen, Ole Maaløesvej 5, DK-2200 Copenhagen, Denmark. ${ }^{5}$ Department of Systems Biology, Center for Biological Sequence Analysis, Technical University of Denmark, Kemitorvet, Building 208, DK-2800 Lyngby, Denmark. ${ }^{6}$ Neuroinflammation Unit, BRIC, University of Copenhagen, Ole Maaløesvej 5, DK-2100 Copenhagen, Denmark. Department of Pathology, Center of Diagnostic Investigation, Rigshospitalet, Blegdamsvej 9, DK-2100 Copenhagen, Denmark. ${ }^{8}$ Brain Tumor Biology Group, Danish Cancer Society Research Center, Strandboulevarden 49, DK-2100 Copenhagen, Denmark. ${ }^{9}$ Department of Oncology, The Finsen Center, Rigshospitalet, Blegdamsvej 9, DK-2100 Copenhagen, Denmark. ${ }^{10}$ Phase I Unit, The Finsen Center, Rigshospitalet, Blegdamsvej 9, DK-2100 Copenhagen, Denmark.

Received: 5 October 2016 Accepted: 31 March 2017

Published online: 18 April 2017

\section{References}

1. Stupp R, Mason WP, van den Bent MJ, Weller M, Fisher B, Taphoorn MJ, et al. Radiotherapy plus concomitant and adjuvant temozolomide for glioblastoma. N Engl J Med. 2005;352:987-96.

2. Seystahl K, Wick W, Weller M. Therapeutic options in recurrent glioblastoma-An update. Crit Rev Oncol Hematol. 2016;99:389-408. (PMID: 26830009)

3. Batchelor $\Pi$, Gerstner ER, Emblem KE, Duda DG, Kalpathy-Cramer J, Snuderl $M$, et al. Improved tumor oxygenation and survival in 
glioblastoma patients who show increased blood perfusion after cediranib and chemoradiation. Proc Natl Acad Sci U S A. 2013;1 10:19059-64.

4. Lu-Emerson C, Duda DG, Emblem KE, Taylor JW, Gerstner ER, Loeffler JS, et al. Lessons from anti-vascular endothelial growth factor and anti-vascular endothelial growth factor receptor trials in patients with glioblastoma. J Clin Oncol. 2015:33:1197-213.

5. Wick W, Brandes AA, Gorlia T, Bendszus M., Sahm F., Taal W. et al. Phase III Trial Exploring the Combination of Bevacizumab and Lomustine in Patients with first Recurrence of a Glioblastoma: The EORTC 26101 Trial. J Clin Oncol. 2016;34. (suppl; abstract 2001).

6. Henriksson R, Asklund T, Poulsen HS. Impact of therapy on quality of life, neurocognitive function and their correlates in glioblastoma multiforme: a review. J Neuro-Oncol. 2011;104:639-46.

7. Huang RY, Rahman R, Ballman KV, Felten SJ, Anderson SK, Ellingson BM, et al. The Impact of T2/FLAIR Evaluation per RANO Criteria on Response Assessment of Recurrent Glioblastoma Patients Treated with Bevacizumab. Clin Cancer Res. 2015;22(3):575-81. (PMID: 26490307).

8. Moller S, Grunnet K, Hansen S, Schultz H, Holmberg M, Sorensen M, et al. A phase II trial with bevacizumab and irinotecan for patients with primary brain tumors and progression after standard therapy. Acta Oncol. 2012:51:797-804

9. Urup T, Dahlrot RH, Grunnet K, Christensen IJ, Michaelsen SR, Toft A, et al. Development and validation of a prognostic model for recurrent glioblastoma patients treated with bevacizumab and irinotecan. Acta Oncol. 2016;55(4):41822. (PMID: 26828563)

10. Erdem-Eraslan L, van den Bent MJ, Hoogstrate Y, Naz-Khan H, Stubbs A, van der Spek $P$, et al. Identification of patients with recurrent Glioblastoma who may benefit from combined Bevacizumab and CCNU therapy: a report from the BELOB trial. Cancer Res. 2016;76:525-34.

11. Sandmann T, Bourgon R, Garcia J, Li C, Cloughesy T, Chinot OL, et al. Patients with Proneural Glioblastoma may derive overall survival benefit from the Addition of Bevacizumab to first-line radiotherapy and Temozolomide: retrospective analysis of the AVAglio trial. J Clin Oncol. 2015;33:2735-44

12. Laffaire J, Di Stefano AL, Chinot O, Idbaih A, Gallego Perez-Larraya J, Marie Y, et al. An ANOCEF genomic and transcriptomic microarray study of the response to irinotecan and bevacizumab in recurrent glioblastomas. Biomed Res Int. 2014;2014:282815.

13. Urup T, Michaelsen SR, Olsen LR, Toft A, Christensen IJ, Grunnet K, et al. Angiotensinogen and HLA class II predict bevacizumab response in recurrent glioblastoma patients. Mol Oncol. 2016;10(8):1160-8. (PMID: 27262894).

14. de Groot JF, Fuller G, Kumar AJ, Piao Y, Eterovic K, Ji Y, et al. Tumor invasion after treatment of glioblastoma with bevacizumab: radiographic and pathologic correlation in humans and mice. Neuro-Oncology. 2010;12:233-42.

15. DeLay M, Jahangiri A, Carbonell WS, Hu YL, Tsao S, Tom MW, et al. Microarray analysis verifies two distinct phenotypes of glioblastomas resistant to antiangiogenic therapy. Clin Cancer Res. 2012;18:2930-42.

16. Keunen O, Johansson M, Oudin A, Sanzey M, Rahim SA, Fack F, et al. Anti-VEGF treatment reduces blood supply and increases tumor cell invasion in glioblastoma. Proc Natl Acad Sci U S A. 2011;108:3749-54.

17. Lu KV, Chang JP, Parachoniak CA, Pandika MM, Aghi MK, Meyronet D, et al. VEGF inhibits tumor cell invasion and mesenchymal transition through a MET/NEGFR2 complex. Cancer Cell. 2012;22:21-35.

18. Piao Y, Liang J, Holmes L, Henry V, Sulman E, de Groot JF. Acquired resistance to anti-VEGF therapy in glioblastoma is associated with a mesenchymal transition. Clin Cancer Res. 2013;19:4392-403.

19. Campos B, Olsen LR, Urup T, Poulsen HS. A comprehensive profile of recurrent glioblastoma. Oncogene. 2016;35(45):5819-25.

20. Poulsen HS, Grunnet K, Sorensen M, Olsen P, Hasselbalch B, Nelausen K, et al. Bevacizumab plus irinotecan in the treatment patients with progressive recurrent malignant brain tumours. Acta Oncol. 2009;48:52-8.

21. Wen PY, Macdonald DR, Reardon DA, Cloughesy TF, Sorensen AG, Galanis E, et al. Updated response assessment criteria for high-grade gliomas: response assessment in neuro-oncology working group. J Clin Oncol. 2010;28:1963-72.

22. Bolger AM, Lohse M, Usadel B. Trimmomatic: a flexible trimmer for Illumina sequence data. Bioinformatics. 2014;30:2114-20.

23. Trapnell C, Pachter L, Salzberg SL. TopHat: discovering splice junctions with RNA-Seq. Bioinformatics. 2009;25:1105-11.

24. Liao Y, Smyth GK, Shi W. featureCounts: an efficient general purpose program for assigning sequence reads to genomic features. Bioinformatics. 2014;30:923-30.
25. Liberzon A, Subramanian A, Pinchback R, Thorvaldsdottir $H$, Tamayo $P$, Mesirov JP. Molecular signatures database (MSigDB) 3.0. Bioinformatics. 2011;27:1739-40.

26. Kramer A, Green J, Pollard Jr J, Tugendreich S. Causal analysis approaches in ingenuity pathway analysis. Bioinformatics. 2014;30:523-30.

27. Verhaak RG, Hoadley KA, Purdom E, Wang V, Qi Y, Wilkerson MD, et al. Integrated genomic analysis identifies clinically relevant subtypes of glioblastoma characterized by abnormalities in PDGFRA, IDH1, EGFR, and NF1. Cancer Cell. 2010;17:98-110.

28. Sitohy B, Nagy JA, Dvorak HF. Anti-VEGF/NEGFR therapy for cancer: reassessing the target. Cancer Res. 2012;72:1909-14.

29. Pereira JD, Sansom SN, Smith J, Dobenecker MW, Tarakhovsky A, Livesey FJ. Ezh2, the histone methyltransferase of $\mathrm{PRC2}$, regulates the balance between self-renewal and differentiation in the cerebral cortex. Proc Natl Acad Sci U S A. 2010;107:15957-62

30. Phillips HS, Kharbanda S, Chen R, Forrest WF, Soriano RH, Wu TD, et al. Molecular subclasses of high-grade glioma predict prognosis, delineate a pattern of disease progression, and resemble stages in neurogenesis. Cancer Cell. 2006;9:157-73.

31. Joseph JV, Conroy S, Tomar T, Eggens-Meijer E, Bhat K, Copray S, et al. TGF-beta is an inducer of ZEB1-dependent mesenchymal transdifferentiation in glioblastoma that is associated with tumor invasion. Cell Death Dis. 2014;5:e1443.

32. Kalluri R, Weinberg RA. The basics of epithelial-mesenchymal transition. J Clin Invest. 2009;119:1420-8.

33. Goel $\mathrm{S}$, Duda DG, Xu L, Munn LL, Boucher Y, Fukumura D, et al. Normalization of the vasculature for treatment of cancer and other diseases. Physiol Rev. 2011;91:1071-121.

34. Huang Y, Goel S, Duda DG, Fukumura D, Jain RK. Vascular normalization as an emerging strategy to enhance cancer immunotherapy. Cancer Res. 2013;73:2943-8.

35. Kaur B, Khwaja FW, Severson EA, Matheny SL, Brat DJ, Van Meir EG. Hypoxia and the hypoxia-inducible-factor pathway in glioma growth and angiogenesis. Neuro-Oncology. 2005;7:134-53.

36. Varadan V, Kamalakaran S, Gilmore H, Banerjee N, Janevski A, Miskimen KL, et al. Brief-exposure to preoperative bevacizumab reveals a TGF-beta signature predictive of response in HER2-negative breast cancers. Int $J$ Cancer. 2016;138:747-57.

37. Yoshiji H, Kuriyama S, Ways DK, Yoshii J, Miyamoto Y, Kawata M, et al. Protein kinase $C$ lies on the signaling pathway for vascular endothelial growth factor-mediated tumor development and angiogenesis. Cancer Res. 1999:59:4413-8.

\section{Submit your next manuscript to BioMed Central and we will help you at every step:}

- We accept pre-submission inquiries

- Our selector tool helps you to find the most relevant journal

- We provide round the clock customer support

- Convenient online submission

- Thorough peer review

- Inclusion in PubMed and all major indexing services

- Maximum visibility for your research

Submit your manuscript at www.biomedcentral.com/submit
) Biomed Central 